\title{
Endoscopic Identification and Removal of an Unusual Symptomatic Colonic Foreign Body
}

\author{
J. RONALD OEHLER, MD, THOMAS L. DENT, MD, MOSTAFA A.H. IBRAHIM, MD, and \\ WILLIAM A. GRACIE, JR, MD
}

Foreign bodies in the gastrointestinal tract can be troublesome and occasionally life threatening. Surgical or endoscopic intervention is indicated when serious complications occur. Greater facility with flexible fiberoptic endoscopes has decreased the need for surgical intervention. In this report, the use of a fiberoptic colonoscope to remove a life threatening foreign body from the transverse colon is described.

Rigid esophagoscopes and sigmoidoscopes have long provided the means for locating and recovering foreign bodies from the esophagus, proximal stomach (1), and distal rectosigmoid $(2,3)$. Flexible fiberoptic endoscopes were initially thought to be of only limited use for retrieving foreign bodies since the only lumen through which foreign bodies could be passed was the tiny biopsy forceps channel. Recently however, a number of reports have appeared which describe the removal of a variety of foreign objects including a sewing needle (4), an open safety pin (5), rubber catheters and knotted nasogastric tubes $(6,7)$, a toothbrush (8), a toothpick (9), pieces of coat hanger wire (10), a 5-centimeter hat pin (11), and a chicken bone (12), as well as an artichoke heart, a prune pit, a hypodermic needle, a thermometer, and a gallstone (13), from the upper gastrointestinal tract with flexible fiberoptic endoscopes. Such reports provide con-

From the Department of Surgery, Section of General Surgery, and the Department of Medicine, Section of Gastroenterology, University of Michigan Medical Center, Ann Arbor, Michigan 48109.

Address for reprint requests: Dr. Thomas L. Dent, Department of Surgery, University of Michigan Medical Center, Ann Arbor, Michigan 48109. vincing evidence that fiberoptic endoscopy can be highly effective in locating and removing foreign bodies from the upper gastrointestinal tract. Reports of foreign body retrieval from regions of the lower gastrointestinal tract which can not be reached by the rigid sigmoidoscope are relatively rare. One reason for this may be that ingested foreign bodies which successfully negotiate the small bowel usually pass through the colon without complication $(2,14)$ while objects which are inserted through the anus seldom ascend higher than the lower rectosigmoid and are thus usually accessable to a rigid sigmoidoscope (2). Reports of transanally introduced objects which have been retrieved from as high in the colon as the hepatic flexure with the aid of a fiberoptic colonoscope $(15,16)$ indicate that fiberoptic endoscopic procedures are effective in removing foreign bodies from the colon as well as from the upper gastrointestinal tract.

In this report we describe the endoscopic removal from the transverse colon of a life-threatening foreign body which was neither ingested orally nor inserted rectally and which could not have been passed spontaneously.

\section{CASE REPORT}

A 30-year-old white male presented with bright red rectal bleeding. He had been in his usual state of good health until several hours prior to admission when, after drinking several beers, he noted lower abdominal cramping and had several bowel movements of formed stool. Shortly thereafter he passed a large amount of bright red blood per rectum. One hour later, the patient again noted lower abdominal cramping and passed an estimated $200-300 \mathrm{ml}$ of bright red blood per rectum. The patient denied the use 


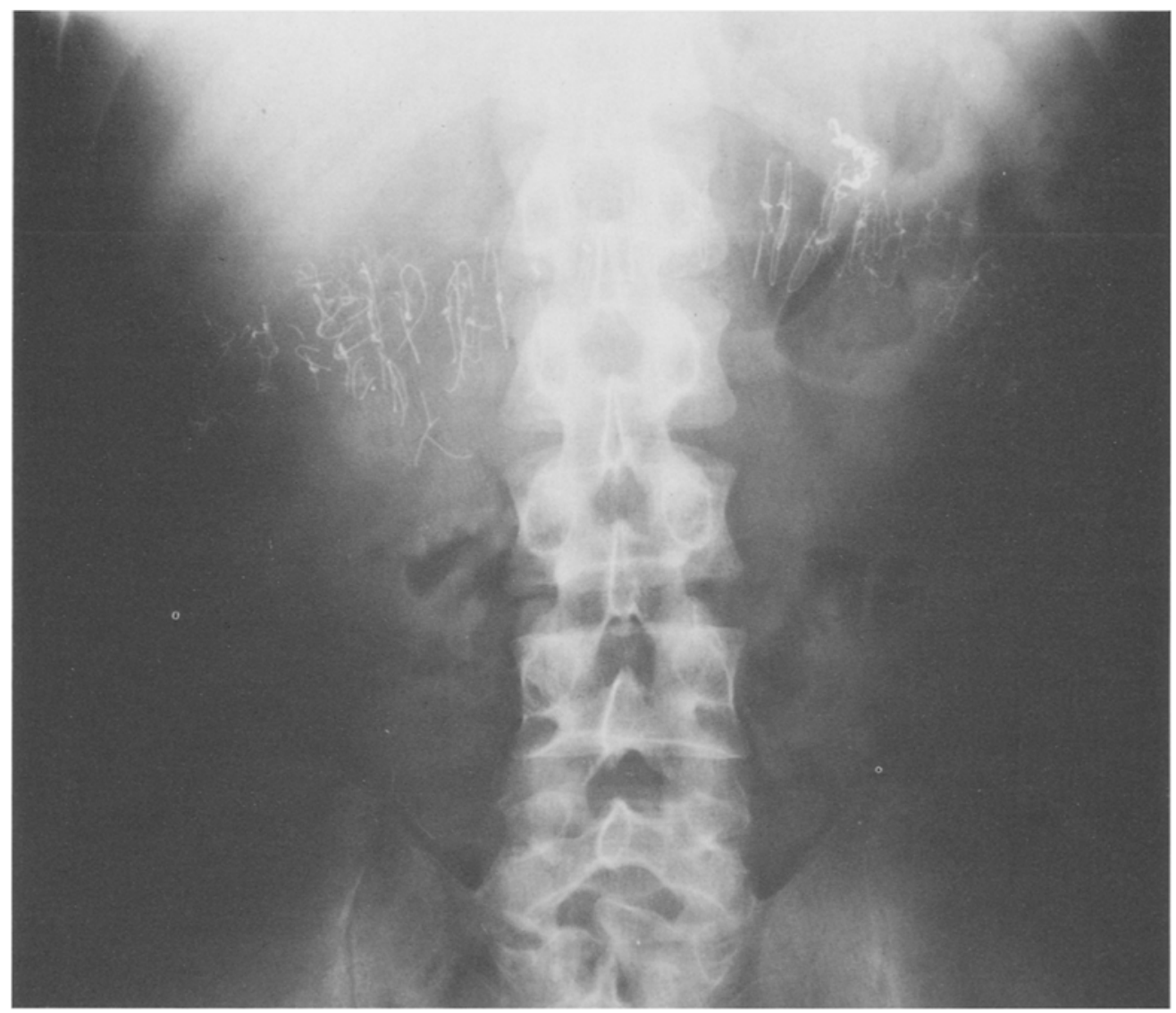

Fig 1. Abdominal $x$-ray obtained on admission revealed numerous wire sutures used in the closure of the abdominal wall. The examination was otherwise normal.

of aspirin or other drugs. Eight years prior to admission, he underwent vagotomy and antrectomy with a Billroth I gastroduodenal anastomosis for a bleeding gastric ulcer and 6 years prior to admission, he sustained injuries in a motor vehicle accident and required an emergency laparotomy in which splenectomy and right adrenalectomy were performed and a hepatic laceration was sutured. Both operations were performed through an extended left subcostal incision. The abdomen was closed in layers with a series of interrupted wire sutures in both cases. Neither operation was complicated and the patient's ulcer disease had not recurred. On physical examination, the patient's vital signs were relatively stable with a heart rate of 90-110 and a blood pressure of 100/50 in the sitting position. The abdomen was soft and nontender with hyperactive bowel sounds. Rectal examination revealed only the presence of bright red blood. Laboratory tests obtained on admission revealed a hematocrit of $39.1 \%$ with a WBC of 8600 cells $/ \mathrm{mm}^{3}$. His prothrombin time was $67 \%$, and his partial thromboplastin time was $47 \mathrm{sec}$ with a control of $35 \mathrm{sec}$. His platelet count was 255,000 . Liver enzymes were mildly elevated with an alkaline phosphatase of 251, an SGOT of 178, and a SGPT of 67. Total bilirubin was 1.3. Admission chest $x$-ray was normal. Abdominal $\mathrm{x}$-ray, obtained on admission, revealed the presence of a large amount of wire suture material, apparently used in the closure of the abdominal incision after the patient's previous operations as mentioned above (Figure 1). The patient was admitted to the intensive care unit and intravenous fluid therapy was begun. Gastric lavage with iced saline revealed no evidence for gross or occult blood. Three and one half hours after admission a repeat hematocrit was $25.2 \%$. Emergency sigmoidoscopy revealed numerous blood clots in the rectosigmoid and rectum but no source of bleeding was found. Emergency esophagogastroduodenoscopy failed to identify the source of bleeding. On the day of admission, the patient received two units of packed cells. On the second hospital day, the patient underwent upper-gastrointestinal barium-contrast studies which only showed evidence of his previous surgery. A Meckel's scan was normal. Later on the second hospital day, the patient again began passing large quantities of bright red blood per rectum and by the third hospital day, he had required transfusion of a total of 6 units of blood and 2 units of fresh frozen plasma to maintain his blood pressure and a hematocrit in the mid-30s. The mas- 

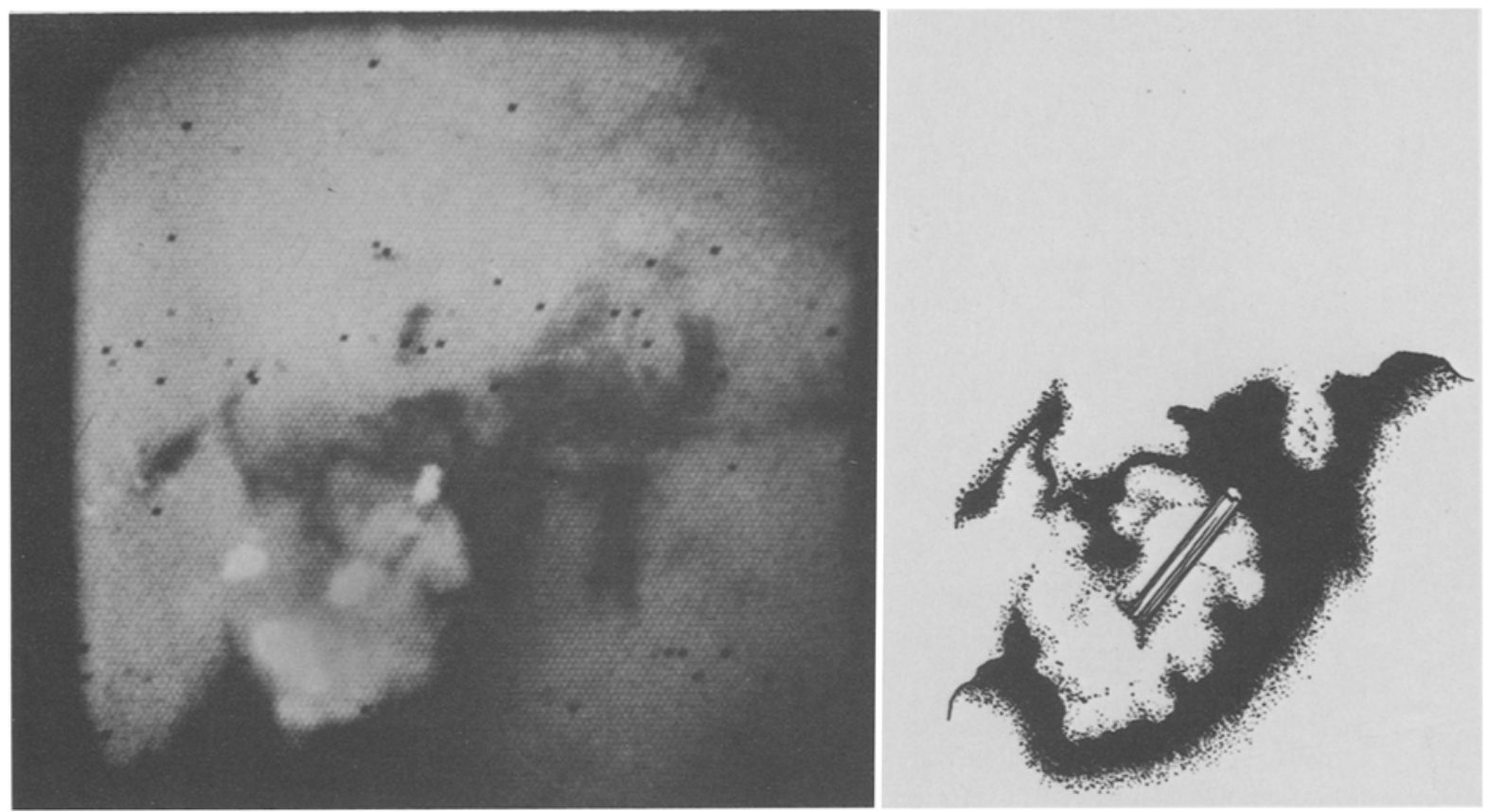

Fig 2. Left: Colonoscopic view of the wire protruding into the colon lumen. Right: Artist's drawing made from a projection of the original slide.

sive bleeding finally stopped spontaneously although guiac-positive stools continued to be recorded daily. On the 8th hospital day, the patient underwent colonoscopy. There was no evidence of inflammatory bowel disease or mass lesions and no hemangiomas were identified. However, in the distal transverse colon, at a distance of $60 \mathrm{~cm}$ from the anal verge, a 5-mm circular area of friable granulation tissue was identified from the center of which protruded a metallic object with a size and shape consistant with that of a broken wire suture (Figure 2). The lesion was slowly oozing blood and when touched with the tip of the colonoscope, considerable bleeding resulted. The colonoscope was carefully withdrawn and the patient was placed on a liquid diet and a regimen of cathartics in preparation for elective colonoscopic removal and possible celiotomy. Colonoscopy was again performed and the lesion described at the time of the orginal examination was visualized. The area was carefully irrigated in order to gain a better view of the wire and to access the possibility of removing it with the colonoscope. Minor hemorrhage resulted from manipulation of the object with the colonoscope tip. Finally, the biopsy forceps were used to grasp the metallic object and with traction, the wire was pulled from its bed. No significant hemorrhage occurred. The wire was held with the biopsy forceps in the center of the colonic lumen while the colonoscope, forceps, and wire was carefully withdrawn under direct vision as a unit in order to prevent injury to the colonic mucosa. The foreign body (Figure 3) was a broken wire suture. The patient was observed, and without signs of recurrent gastrointestinal hemorrhage, was discharged the following day. He has now been followed for 6 months with no evidence of recurrent gastrointestinal bleeding.

\section{DISCUSSION}

Fiberoptic endoscopy has been shown to be useful in the removal of foreign bodies from the upper gastrointestinal tract $(4-14,17)$. The successful snare removal of colon polyps via the colonoscope $(17,18$, and review in 19) suggests that colonoscopes can be used to remove foreign bodies from the colon. Colonoscopists, however, are infrequently called upon to retrieve foreign objects from the colon because objects inserted through the anus seldom lodge in the colon higher than the reach of the sigmoidoscope (2) and ingested foreign bodies usually pass through the colon without complication after traversing the small bowel (14). Our patient is unique in that his foreign body was neither ingested nor inserted per rectum, was highly unlikely to be passed per rectum and further, represented a threat to the patient's life. Review of the report of the patient's original operation revealed that when the patient was extubated at the end of the operation, the abdominal wall closure became disrupted and an immediate reexploration and operative repair of the defect with additional wire suture material was required. It seems likely that one of the broken wire sutures eventually migrated through the area of the incision and eroded the colonic mucosa, resulting in the sudden onset of brisk bright red rec- 


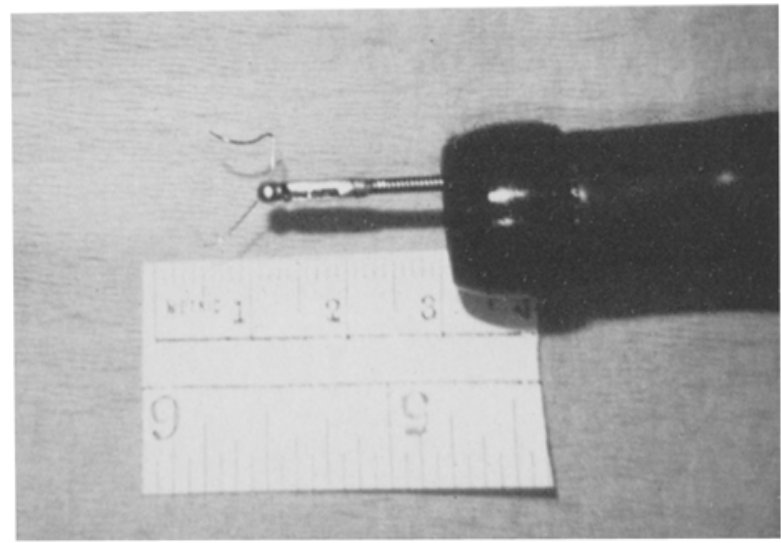

Fig 3. Colonoscopic tip and biopsy forceps holding the broken wire suture just after removal from the patient's transverse colon.

tal bleeding. Endoscopic removal of the wire suture spared the patient an operation and a bowel resection.

\section{SUMMARY}

The discovery and removal of a life-threatening colonic wire suture using the flexible fiberoptic colonoscope has been described. Such reports demonstrate the versatility and usefulness of diagnostic and therapeutic endoscopic procedures.

\section{REFERENCES}

1. Jackson C, Jackson CL: Diseases of the Air and Food Passages of Foreign Body Origin. W.B. Saunders, Philadelphia, 1937

2. Beall AC, DeBakey ME: Injuries and foreign bodies of the colon and rectum. Diseases of the Colon and Rectum. W.B. Saunders, Philadelphia, 1969, p. 872

3. Haft JS, Benjamin HW, Wagner M: Vaginal vibrator lodged in rectum. Br J Med 1:626-628, 1976

4. Gelzayd EA, Jetly J: Fiberendoscopy: Removal of a retained sewing needle from the stomach. Gastrointest Endosc 18:161-165, 1972

5. Grișold C, Haislip CE, Gardner RJ: Removal of an intragastric foreign body using the flexible fiberoptic esophagoscope. Gastrointest Endose 19:194-200, 1973

6. Maimon HN, Mulligan FD: Removal of a foreign body from the stomach. Gastrointest Endose 18:163-170, 1972
7. Graham DY, Schwartz JT: Endoscopic removal of a knotted feeding tube from an infant. Gastrointest Endosc 21:32-33, 1974

8. Nacianceno SE, Joseph RR: Therapeutic endoscopy: Removal of an unusual foreign object from the stomach. Gastrointest Endose 21:34, 1974

9. Darby JP: Fiberendoscopic removal of a non-radiopaque foreign body (toothpick) from the stomach. Gastrointest Endose 21:34-35, 1974

10. Kline MM: Endoscopic snare in removal of an esophageal foreign body. Gastrointest Endosc 20(4):165-166, 1974

11. DeGerome JH: Snare extraction of a gastric foreign body. Gastrointest Endosc 20(4):73-74, 1973

12. McCaffery TD, Lilly JO: The management of foreign affairs of the GI tract. Am J Dig Dis 20(2):121-126, 1975

13. Olsen H, Lawrence W, Berstein R: Fiberendoscopic removal of foreign bodies from the upper gastrointestinal tract. A simple and consistent method using a snare. Gastrointest Endosc 21(2):58-60, 1974

14. Stevenson EO, Hastings N: Foreign bodies in gastrointestinal tract of infants and children. Am Surg 34:151-158, 1968

15. Wolf L, Geraci K: Colonscopic removal of balloons from the bowel. Gastrointest Endosc 24(1):41-44, 1977

16. Richter RM, Littman L: Endoscopic extraction of an unusual colonic foreign body. Gastrointest Endosc 22(1):40-45, 1971

17. Wolff WI, Shinya H: Colonofiberoscopy. JAMA 217:1509, 1971

18. Wolff WI, Shinya H: Polypectomy via the fiberoptic colonoscopy. N Engl J Med 288:329-332, 1973

19. Marks G, Moses ML: The clinical application of flexible fiberoptic colonoscopy. Surg Clin N Am 53(3):735-756, 1973 